\title{
Study Site Protocol Version Relationship
}

National Cancer Institute

\section{Source}

National Cancer Institute. Study Site Protocol Version Relationship. NCI Thesaurus. Code C93472.

Specifies the link between a study site and a version of the study protocol used or available for use at that site. 